\title{
The discoid medial meniscus: Pitfalls and review of the literature
}

\author{
Jlalia $Z^{1,2 *}$, Maatoug $M^{1}$, Ben Ahmed $S^{1}$, Kammoun $K^{1}{ }^{1}$ and Jenzri $M^{1}$ \\ ${ }^{1}$ Department of pediatric orthopedics, Kassab institute, Kasar said, Tunisia \\ ${ }^{2}$ Faculty of Medicine of Tunisia, University of Tunisia El Manar, Tunisia
}

\begin{abstract}
The discoid meniscus is a rare meniscal abnormality. Through this case of a patient with internal discoid meniscus, we propose to study that malformation, clinical and radiological presentation and therapeutic modalities. We report the case of the child 5 years old, who visited emergency with a pain in left knee following a trauma. The clinical examination showed a meniscal Syndrome. MRI showed a lesion bucket handle medial meniscus without any morphological abnormality of the meniscus. The exploration of the knee joint by arthroscopy showed an incomplete discoid medial meniscus with the presence of a wide bucket handle injury dislocated in intercondylar space. The patient underwent resection of the dislocated part with adjustment of the axial edge of the medial meniscus realizing an arciform shape similar to the normal meniscal form. The functional result after five years was excellent.
\end{abstract}

\section{Introduction}

Discoid Meniscus (MD) is a rare meniscal abnormality. Although it is usually located in the lateral side compartment, it may be more rarely medial. Etiopathogenesis is not known but congenital etiology is most likely. The MD is usually asymptomatic. Magnetic resonance imaging (MRI) can provide accurate diagnosis and help preoperative planning. We report the case of a patient who presented a discoid medial meniscus of perioperative Fortuitous discovery during arthroscopy for cure of a dislocated bucket loop injury in the notch. This malformation was not visualized by MRI done preoperatively. The bucket handle injury resulted in a kind of natural saucer which returned to the meniscus its normal arciform shape.

\section{Case report}

We report the case of the 5 years old boy with no significant pathological history who consulted our emergencies for a painful stiffness of the left knee following a 15 days after trauma. The child was apyretic, the knee was swollen with the presence of a patellar shock. The palpation of internal articular interlining is painful. There was a knee reducible flessum of $30^{\circ}$. The standard radiography showed a discreet enlargement of the internal articular interlining (Figure 1). MRI showed a bucket handle injury of the internal meniscus (Figure 2). A partial meniscectomy under arthroscopy was performed. In peroperative we discovered an incomplete discoid medial meniscus (type II Watanabe), with presence of an extended lesion of the medial meniscus. The patient had a resection of the dislocated part with regularization of the axial margin of the internal meniscus, thus producing an arched shape similar to the normal shape. The operative sequences were simple. The patient is asymptomatic until now at 5 years of follow-up. The functional result was excellent with complete knee mobility and normal sports activity.

\section{Discussion}

Discoid Meniscus is a rare anatomical variant where the normal fibrocartilage is replaced by a thickening which can sometimes occur

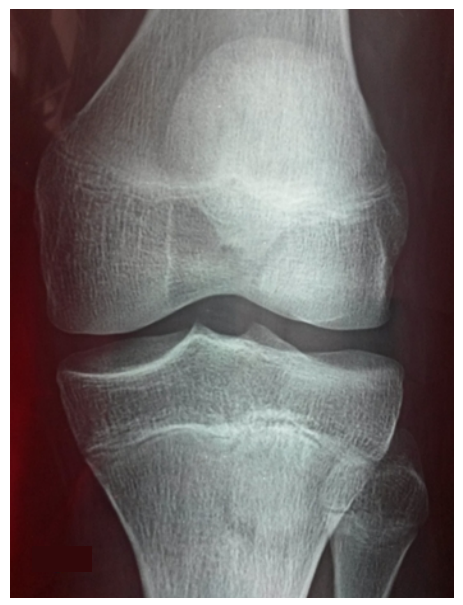

Figure 1. Radiography of the knee of the face: enlargement of the articular space internally

up to the median line of the knee in its complete form [1]. Although it is usually located in the side compartment, it may be rarely medial. The lateral discoidal meniscus was described for the first time by Young in 1889 , and the first medial discoid meniscus was reported by WatsonJones in $1930[2,3]$. The prevalence of the discoid lateral meniscus is $1.5-15.5 \%$, while the highest prevalence of a medial discoid meniscus is $0.3 \%[3,4]$. Watanabe performed the first arthroscopic surgery for discoid meniscus in 1962, he classified the discoid meniscus into three forms: complete, incomplete, and shape with a Wrisberg ligament [4].

${ }^{\star}$ Correspondence to: Zied Jlalia, Department of pediatric orthopedics, Kassab institute for orthopedic surgery, Ksar said 2010 Manouba, Tunisia, E-mail: zied_j@yahoo.fr

Key words: discoid meniscus, MRI, child

Received: November 24, 2018; Accepted: December 10, 2018; Published: December 13, 2018 




Figure 2. MRI of the knee: thickening of the internal meniscus, lesion in a loose pail loop in the intercondylar incision of the knee

Only the complete and incomplete types of discoid medial menisci Have been reported in the literature. The type with the Wrisberg ligament is a variant that has not been identified for the medial discoidal menisci [5]. In 1948, Smillie suggested that in the early stages of development the meniscus existed as a cartilaginous disk and that the discoid meniscus was the result of a stoppage in the development of the meniscus at different stages of embryological growth [6]. In an embryological study in 1957, Kaplan demonstrated that there is no time during the normal embryological development during which the meniscus has a discoidal form [7]. Kaplan suggested an etiopathogenesis influenced by mechanical factors such as a hypermobile posterior segment. $\mathrm{He}$ also described the type "Wrisberg ligament" in his embryological and cadaveric studies [7]. In 1983, Clark and Ogden found that meniscus acquire their semilunar form early during prenatal development [8]. They suggest that the discoid meniscus may be the result of instability due to the absence of the meniscus-femoral attachments. A recent histomorphological study demonstrated discontinuity and heterogeneity of the collagen network in the discoid meniscus compared to the normal meniscus [9]. Such a finding may support the theory that the discoid meniscus represents a structural lesion rather than a morphological variant. Eight cases of abnormal LCA insertion on a medial discoid meniscus have been reported in the literature [1]. An embryological study has shown that at the 10th week of intrauterine development, the cartilage has a shape that is very similar to that of an adult. This study showed that an intermediate mesenchymal plaque or blastema decomposes to form a space between the two bones towards the end of the second month [10]. In their fundamental work of 1901, Bardeen and Lewis stated that the menisci and the cruciate ligament are differentiated directly from this blastema in a sequential order. This led to the conclusion that during embryonic development, probably between the ninth and tenth weeks, there is a separation failure between the medial meniscus and the LCA $[11,12]$

This results in failure of the resorption of the apical part of the internal meniscus which induces a discoid morphology that remains attached to the ACL. Apart from a lesion the MD is often asymptomatic. Zhang reported a case of symptomatic medial discoid meniscus in the absence of any meniscal lesion [13]. In everyday practice, standard radiography was commonly used as a first-line examination. However, its contribution to the diagnosis of discoid menisci has always been poor. MRI can provide accurate diagnosis and help preoperative planning. A meniscus is discoidal when a continuity of the meniscus is observed between its anterior and posterior horn on at least 3 contiguous sagittal sections $5 \mathrm{~mm}$ thick. Radiological diagnosis can be sustained by demonstrating a difference in height between the disc meniscus and the normal meniscus greater than or equal to $2 \mathrm{~mm}$ [14]. The literature reports associations with underlying bone changes with increased concavity and depression of the medial tibial plateau, but these signs do not play a decisive role in the diagnosis [9]. Although these changes are not supposed to be specific to the discoid lateral meniscus, this study may provide physicians with tools to assist in the diagnosis of the discoid medial meniscus on standard radiography. In our case, the MRI did not show the discoid shape of the meniscus. This can be explained by the bucket handle nature of the lesion. Indeed, the dislocation of the bucket handle in the indentation camouflaged the discoidal nature of the meniscus and achieved a kind of "natural sausage" of the meniscus thus making it its normal arciform shape. The recommended treatment for the medial discoidal meniscus depends on the morphology of the meniscal lesion which is evident during arthroscopic exploration. Historically, a total meniscectomy was the gold standard because it was believed that discoid meniscus tissue was abnormal $[7,15]$. However, this technique has been shown to accelerate osteoarthritis and has been replaced by partial meniscectomy. Kim et al. described a monobloc arthroscopic excision specific for the treatment of the medial discoid meniscus [16]. Washington et al. showed good long-term results after total disc meniscal meniscus meniscectomy in children after an average follow-up of 17 years [17]. Studies have shown that results after partial meniscectomy were better than those after total meniscectomy for lateral discoid meniscus [17]. In our case, the clinical result after 5 years of recoil was excellent, the child was asymptomatic and the function of the knee was complete. The standard radiograph shows no changes. The partial meniscectomy under arthroscopy associated with the meniscal repair of the peripheral lesion in young patients has demonstrated its efficacy in the treatment of the lateral discoidal meniscus [18]. It can eventually decrease the development of osteoarthritis over time. However, a cadaveric study showed that normal perimeniscal attachments are strong and complex, while peripheral attachments of the discoid meniscus are often relatively small, which is why most discoid meniscus have been treated with total meniscectomy [18].

\section{Conclusion}

The medial discoid meniscus is an extremely rare anomaly. Clinical signs and symptoms of internal discoid meniscal lesions are similar to those of any other meniscal lesion. No Wrisberg ligament was found in the medial discoidal meniscus. Meniscectomy is the main method of treatment of medial meniscal lesions. The evolution towards osteoarthritis of the knee remains the main complication of this technique.

\section{References}

1. Michael H (2011) Horizontal cleavage tear of discoid medial meniscus diagnosed on MRI and treated with arthroscopic partial resection. Knee Surg Sports Traumatol Arthrosc 19: 1860-1867. [Crossref]

2. Young RB (1889) The external semilunar cartilage as a complete disc. Williams and Norgata 179.

3. Watson-Jones R (1930) Specimen of internal semilunar cartilage as a complete disc. Proc R Soc Med 23: 1588-1589. [Crossref]

4. Jeannopoulos CL (1950) Observations on discoid menisci. J Bone Jt Surg Am 32: 649 652. [Crossref]

5. Papadopoulos A, Karathanasis A, Kirkos JM, Kapetanos GA (2009) Epidemiologic, clinical and arthroscopic study of the discoid meniscus variant in Greek population. Knee Surg Sports Traumatol Arthrosc 17: 600-606. [Crossref] 
6. Smillie IS (1948) The congenital discoid meniscus. J Bone Jt Surg Br 30: 671-682. [Crossref]

7. Kaplan EB (1957) Discoid lateral meniscus of the knee joint: nature, mechanism, and operative treatment. J Bone Jt Surg Am 39: 77-87. [Crossref]

8. Clark CR, Ogden JA (1983) Development of the menisci of the human knee joint. Morphological changes and their potential role in childhood meniscal injury. $J$ Bone Joint Surg Am 65: 538-547. [Crossref]

9. Papadopoulos A, Kirkos JM, Kapetanos GA (2009) Histomorphologic study of discoid meniscus. Arthroscopy 25: 262-268. [Crossref]

10. Sorren A (1985) On the etiology of congenital malformation of the meniscus. Arch Orthop Trauma Surg 104: 283-288. [Crossref]

11. Bardeen CR, Lewis WH (1901) Development of the limbs, body wall and back in man Am J Anat 1:1.

12. Min BH, Ha HK, Khang SY (2001) Medial discoid meniscus completely coalesced with the anterior cruciate ligament. Arthroscopy 17: E27. [Crossref]
13. Zhang C, Xu H, Wang Y (2013) Symptomatic discoid medial meniscus. J Orthop Sports Phys Ther 43: 428

14. Silverman JM, Mink JH, Deutsch AL (1989) Discoid menisci of the knee: MR imaging appearance. Radiology 173: 351-354

15. Aichroth PM, Patel DV, Marx CL (1991) Congenital discoid lateral meniscus in children. A following study and evolution of management. J Bone Joint Surg Br 73 : 932-936. [Crossref]

16. Kim SJ, Kwun JD, Jung KA, Kim JM (2005) Arthroscopic excision of the symptomatic discoid medial meniscus in one piece: a surgical technique. Arthroscopy 21: 1515. [Crossref]

17. Dickout SC, Delee JC (1982) The discoid lateral-meniscus syndrome. J Bone Joint Surg Am 64: 1068-1073. [Crossref]

18. Adachi N, Ochi M, Uchio Y, Kuriwaka M, Shinomiya R (2004) Torn discoid lateral meniscus treated using partial central meniscectomy and suture of the peripheral tear Arthroscopy 20: 536-542. [Crossref]

Copyright: @2018 Jlalia Z. This is an open-access article distributed under the terms of the Creative Commons Attribution License, which permits unrestricted use, distribution, and reproduction in any medium, provided the original author and source are credited. 